livraisons

d'Histoire

de l'Architecture

\section{Livraisons de l'histoire de l'architecture}

32 | 2016

Les représentations de l'architecture

\title{
La transformation du château de Versailles par Louis-Philippe (1833-1847) en coulisses : les rapports des visites royales de l'architecte Frédéric Nepveu
}

Isabelle Chave et Éric Landgraf

\section{(2) OpenEdition \\ Journals}

Édition électronique

URL : http://journals.openedition.org/lha/645

DOI : 10.4000/lha.645

ISSN : 1960-5994

Éditeur

Association Livraisons d'histoire de l'architecture - LHA

Édition imprimée

Date de publication : 31 décembre 2016

Pagination : 127-145

ISSN : $1627-4970$

Référence électronique

Isabelle Chave et Éric Landgraf, « La transformation du château de Versailles par Louis-Philippe

(1833-1847) en coulisses : les rapports des visites royales de l'architecte Frédéric Nepveu », Livraisons de l'histoire de l'architecture [En ligne], 32 | 2016, mis en ligne le 31 décembre 2018, consulté le 19 avril 2019. URL : http://journals.openedition.org//ha/645 ; DOI : 10.4000/lha.645 


\section{LA TRANSFORMATION DU CHÂTEAU DE VERSAILLES PAR LOUIS-PHILIPPE (1833-1847) EN COULISSES : LES RAPPORTS DES VISITES ROYALES DE L'ARCHITECTE FRÉDÉRIC NEPVEU}

Les Archives nationales ont acquis en octobre 2009, par voie de revendication lors d'une vente aux enchères organisée par une société de vente d'autographes, et réintégré au fonds de l'Intendance générale de la Liste civile sous la monarchie de Juillet (sous-série O/4) quatre registres de comptes rendus, par Frédéric Nepveu, architecte du château de Versailles ${ }^{1}$, des visites de chantier menées par le roi LouisPhilippe, de 1833 à 1847, en vue de la création des galeries historiques de l'Histoire de France. Ces rapports techniques reflètent la volonté du roi Louis-Philippe, au fil de ses visites, de transformer les salles du château de Versailles et du palais du Grand Trianon.

À l'occasion de leur numérisation et de leur récente mise en ligne, associée à la réalisation d'un inventaire détaillé consultable en salle des inventaires virtuelle ${ }^{2}$, il est apparu intéressant, d'une part, de confronter ces volumes, de manière plus approfondie au plan diplomatique, aux deux autres exemplaires connus, conservés aux châteaux de Versailles ${ }^{3}$ et de Chantilly ${ }^{4}$, et, d'autre part, de les replacer dans la chaîne administrative, et donc archivistique, des travaux aux résidences royales menés par le roi Louis-Philippe sur les crédits de sa Liste civile.

1. Lire notamment Albert Terrade, "Frédéric Nepveu, architecte du roi Louis-Philippe, anecdotes et souvenirs ", Mémoires de la Société des sciences morales, des lettres et des arts de Seine-et-Oise, 1894, t. XVIII ; Valérie Bajou, "Frédéric Nepveu et le rez-de-chaussée de l'aile du Midi, première campagne de travaux de Louis-Philippe à Versailles ", Gazette des Beaux-Arts, 1999, no 133, 1564-1565, p. 239-270 ; Émilie Biraud, "Frédéric Nepveu (1777-1862), l'architecte et ses réalisations dans le corps central du château de Versailles, 1833-1848 ", mémoire de recherche de l'École du Louvre, 2008 ; de la même, "Frédéric Nepveu (1777-1862) : personnalité d'un architecte dans l'ombre du château de Versailles ", Livraisons de l'histoire de l'architecture, 18|2009 [http://lha.revues.org/221], et "Frédéric Nepveu, architecte du château de Versailles sous la monarchie de Juillet ", Versailles: ville, architecture et nature. Revue de l'histoire de Versailles et des Yvelines, t. 92, 2010, p. 118-139.

2. Les vues, numérisées en 2011 , de ces quatre registres cotés $\mathrm{O} / 4 / 2857$ à O/4/2860 aux Archives nationales (site de Pierrefitte-sur-Seine), sont accessibles à partir de leur inventaire détaillé des rapports et plans reliés: Galeries historiques du château de Versailles (1833-1847), inventaire des rapports de visites du roi Louis-Philippe et des plans numérisés, par Isabelle Chave et Éric Landgraf, Archives nationales, 2016 [https ://www.siv.archives-nationales.culture.gouv.fr/siv/IR/FRAN_IR_054729].

3. Château de Versailles, département des Ressources documentaires, service des Archives, B 27 a-d.

4. Château de Chantilly, service Bibliothèques et Archives, ms. 1349 à ms. 1352 ("Restauration du château de Versailles ", registres de F. Nepveu). 


\section{Les registres Frédéric Nepveu des Archives nationales}

Ces "Galeries historiques du Palais de Versailles» des Archives nationales constituent un ensemble de quatre volumes reliés, réunissant les lettres originales adressées du 26 juillet 1833 au 23 janvier 1847 par Frédéric Nepveu (1777-1862) à O. Godard-Dubuc (1790-1873), architecte de formation, directeur des Dépenses des bâtiments de la Couronne au sein de l'Intendance générale de la Liste civile (1832-1848), "contenant les détails de chaque visite du roi Louis-Philippe et le résumé des ordres donnés par le roi pour la création des Galeries historiques ", selon le titre manuscrit porté en tête de chaque tome.

Le roi des Français décide, en 1833, de restaurer les appartements royaux et de transformer les autres en musée national consacré à l'Histoire de France et dédié "à toutes les gloires " de la France. Passionné d'histoire, Louis-Philippe veut y rassembler en très grand nombre une iconographie, peinte, sculptée, dessinée ou gravée, propre à illustrer des événements et des personnages de l'Histoire de France depuis ses origines, puisant dans les fonds des anciennes collections royales, princières, privées et institutionnelles et les complétant par des milliers de copies et d'œuvres rétrospectives commandées aux artistes contemporains. Chargé d'organiser le tout, l'architecte Nepveu dut détruire de nombreux appartements princiers, surtout dans les deux grandes ailes du château où ces Galeries historiques sont encore en place aujourd'hui ${ }^{5}$.

Le premier volume (26 juillet 1833-23 janvier 1837) contient 94 lettres, sur 207 feuillets, et 2 feuillets de plans; le deuxième volume (30 janvier 1837 13 octobre 1841), 137 lettres, sur 216 feuillets, et 2 feuillets de plans ; le troisième volume (26 octobre 1841-16 décembre 1843), 80 lettres, sur 182 feuillets, et 22 feuillets de plans en couleur ou dessins hors texte, et le quatrième volume (22 janvier 1844-24 décembre 1847), 100 lettres et 4 feuillets explicatifs de plans, sur 182 feuillets, 19 feuillets de plans et 1 plan en double page en couleur.

Les quatre tomes in-folio sont reliés de maroquin vert, signés, en bas du dos du tome IV, de la marque de la veuve Niedrée; onze filets dorés encadrent les plats; le dos, à six nerfs, est orné de caissons de filets dorés, double filet sur les coupes; les tranches sont dorées et de la dentelle dorée a été placée sur les chasses. Cet exemplaire tient son appellation d' "albums Montalivet " des initiales [CM] au centre des plats supérieurs, du nom de Camille Bachasson, comte de Montalivet (1801-1880). Ces rapports et plans ne firent pas, de fait, partie du versement aux Archives nationales des archives de souveraineté, dites "archives du Louvre", obtenu par le directeur Letrosne à la faveur de la révolution de 1848 et mis en œuvre par Goeschler jusqu'en juillet-août 1849. Comme il était d'usage au $\mathrm{XIX}^{\mathrm{e}}$ siècle, ils furent conservés par l'intendant général de la Maison du Roi ${ }^{6}$ par devers lui, en février 1848, malgré leur caractère indéniable d'archives de fonction.

5. Pierre Francastel, La Création du musée de Versailles et la transformation du palais (1832-1848), Versailles, Léon Bernard, 1930.

6. Intendant provisoire de la dotation de la Couronne, du 10 octobre au 2 novembre 1830, puis intendant général de la Liste civile à deux reprises (10 octobre 1832-22 février 1836 et 30 mars 1839-2 février 1848), en alternance avec le baron Fain, Montalivet fut l'ami et exécuteur testamentaire du roi Louis-Philippe. 
Les deux autres exemplaires connus sont de provenance différente: celui de Versailles, relié dans la seconde moitié du XIX ${ }^{\mathrm{e}}$ siècle, provient de la collection d'Henri Grosseuvre (1865-1943), qui le tenait de Paul Favier, collaborateur de Frédéric Nepveu à l'agence d'architecture du château de Versailles. Celui de Chantilly, relié en veau fauve, est un don de la veuve de Frédéric Nepveu au duc d'Aumale, après la mort de l'architecte en $1862^{7}$.

En vue de leur mise en ligne, destinée à préserver les originaux et à garantir une meilleure diffusion des contenus auprès de la communauté scientifique, les 414 comptes rendus de visite et les 47 documents figurés hors texte, ainsi reliés, ont été décrits dans le détail, incluant le relevé des mentions marginales des rapports, en lien avec leur suivi administratif, et, pour les plans, celui de toutes les inscriptions-marques. Réalisé pour le chantier de Versailles, ce traitement de détail n'a pas d'équivalent pour les autres résidences royales entretenues, remaniées ou restaurées par le roi Louis-Philippe sur les crédits alloués par sa Liste civile; c'est aussi qu'au sein des archives de l'Intendance générale de la Liste civile, aucun ensemble comparable n'est conservé aux Archives nationales pour aucun des autres châteaux royaux. On ne pourra ainsi citer que les émargements du personnel des châteaux royaux ${ }^{8}$, les épaves de dossiers de gestion des travaux dans les bâtiments de la Couronne ${ }^{9}$, épargnés par l'incendie et le pillage des Tuileries en février 1848, et la documentation budgétaire et comptable de la Maison du Roi et de la Liste civile, sauvée par ses versements réguliers, durant la monarchie de Juillet, aux archives de la Couronne, installées au Louvre ${ }^{10}$. Ces mandats de paiement pour l'entretien des bâtiments de la Couronne se trouvent certes enrichis de leurs "attachements ", suites de procès-verbaux de travaux effectués par corps de métiers dans un bâtiment donné, formant, une fois réunis et enliassés, les "mémoires » de travaux, qui consacrent une ligne à chaque dépense et mentionnent la somme finale due à chaque entrepreneur. Mais la conduite même des chantiers aux bâtiments royaux (correspondance, devis, plans...) reste, hors des fonds d'agence, très pauvrement documentée.

7. Catalogue général des manuscrits des bibliothèques publiques de France, Paris, Bibliothèque de l'Institut, 1928, p. 450-451 ("Chantilly, les manuscrits»).

8. Arch. nat., $\mathrm{O} / 4 / 3$ à $\mathrm{O} / 4 / 41 / 2$, Administration des châteaux royaux : émargements du personnel (1830-1848).

9. Arch. nat., $\mathrm{O} / 4 / 2781$ à $\mathrm{O} / 4 / 2787$, Déclarations mensuelles des travaux aux bâtiments de la Couronne: lettres d'envoi, prix global des travaux (1831-1837). Voir l'inventaire de Brigitte Labat-Poussin (1993) en ligne [https://www.siv.archives-nationales.culture.gouv.fr/siv/IR/FRAN_ IR_001672].

10. Arch. nat., $\mathrm{O} / 4 / 1329$ à $\mathrm{O} / 4 / 2375$, Travaux dans les bâtiments de la Couronne payés au titre de la Liste civile de Louis-Philippe (1832-1848). Voir en ligne l'inventaire détaillé de David Frapet et Isabelle Chave (2010-2016), issu du dépouillement des budgets de travaux [https://www.siv.archivesnationales.culture.gouv.fr/siv/IR/FRAN_IR_055429]. 


\section{II. Étude comparative des trois exemplaires connus}

Rédigées par les collaborateurs de l'agence d'architecture de Nepveu, les trois séries de registres conservées présentent plusieurs variantes sensibles : nombre de rapports reliés au total (414 pour l'exemplaire des Archives nationales, 407 pour ceux de Versailles et Chantilly), transitions $1836 / 1837$ et 1843/1844, différences de notes de marge des rapports, degrés divers d'élaboration des croquis d'illustration portés en marge, différences de main entre les divers registres.

Tableau détaillé comparatif des trois exemplaires conservés à Pierrefitte-surSeine, Versailles et Chantilly ${ }^{11}$ :

\begin{tabular}{|l|c|c|c|c|c|c|c|c|c|}
\hline & \multicolumn{2}{|c|}{ Vol. } & \multicolumn{2}{|c|}{ Vol. 2} & \multicolumn{2}{c|}{ Vol. 3 } & \multicolumn{2}{|c|}{ Vol. 4} & Nates \\
\hline $\begin{array}{c}\text { Lieu de } \\
\text { conservation } \\
\text { des lettres }\end{array}$ & $\begin{array}{c}\text { Dates } \\
\text { extrêmes }\end{array}$ & Nbre & $\begin{array}{c}\text { Dates } \\
\text { extrêmes }\end{array}$ & Nbre & $\begin{array}{c}\text { Dates } \\
\text { extrêmes }\end{array}$ & Nbre & $\begin{array}{c}\text { Dates } \\
\text { extrêmes }\end{array}$ & Nbre & $\begin{array}{c}\text { Total } \\
\text { des lettres } \\
\text { conservées }\end{array}$ \\
\hline $\begin{array}{l}\text { Archives } \\
\text { nationales }\end{array}$ & $\begin{array}{c}19 / 06 / 1833 \\
\text { au } \\
22 / 01 / 1837\end{array}$ & 93 & $\begin{array}{c}29 / 01 / 1837 \\
\text { au } \\
7 / 10 / 1841\end{array}$ & 142 & $\begin{array}{c}20 / 10 / 1841 \\
\text { au } \\
15 / 01 / 1844\end{array}$ & 80 & $\begin{array}{c}20 / 01 / 1844 \\
\text { au } \\
10 / 12 / 1847\end{array}$ & 98 & 414 \\
\hline $\begin{array}{l}\text { Château } \\
\text { de Versailles }\end{array}$ & $\begin{array}{c}19 / 06 / 1833 \\
\text { au } \\
12 / 12 / 1836\end{array}$ & 95 & $\begin{array}{c}22 / 01 / 1837 \\
\text { au } \\
12 / 11 / 1840\end{array}$ & 120 & $\begin{array}{c}13 / 01 / 1841 \\
\text { au } \\
15 / 12 / 1843\end{array}$ & 93 & $\begin{array}{c}20 / 01 / 1844 \\
\text { au } \\
10 / 12 / 1847\end{array}$ & 99 & 407 \\
\hline $\begin{array}{l}\text { Château } \\
\text { de Chantilly }\end{array}$ & $\begin{array}{c}19 / 06 / 1833 \\
\text { au } \\
12 / 12 / 1836\end{array}$ & 97 & $\begin{array}{c}22 / 01 / 1837 \\
\text { au } \\
27 / 11 / 1840\end{array}$ & 116 & $\begin{array}{c}13 / 01 / 1841 \\
\text { au } \\
15 / 12 / 1843\end{array}$ & 96 & $\begin{array}{c}20 / 01 / 1844 \\
\text { au } \\
10 / 12 / 1847\end{array}$ & 98 & 407 \\
\hline
\end{tabular}

\section{Teneur et justification des rapports de visite}

Sur la durée du règne, Louis-Philippe effectua 398 visites sur le chantier de Versailles, à fréquence régulière - l'équivalent de plus d'une année entière de son règne, en tenant compte du fait que, y compris les allers-retours et les déplacements in situ, sa mobilisation était de presque une journée à chaque visite -, avec un pic avant l'inauguration de 1837 , date à laquelle il établit sa résidence provisoire à Trianon. Il y mène architectes ${ }^{12}$, membres de sa famille, monarques de passage, généraux, ministres parfois. Selon l'analyse globale des rapports de Frédéric Nepveu, le Roi espace ses venues de trois à dix-huit jours, sauf au mois de janvier, où il met toujours beaucoup de temps à revenir, parfois jusqu'à plus de quarante jours, d'une année sur l'autre.

À l'issue de chaque déplacement, l'architecte produit un rapport notifiant, étape par étape, les travaux nécessaires aux changements souhaités dans les salles du

11. (C) Éric Landgraf, 2016.

12. Lire Pierre-François-Léonard Fontaine, Journal, 1799-1853, Paris, École nationale des Beaux-Arts / Institut français d'architecture / Société d'histoire de l'art français, 1987, 2 vol. 
palais, aux constructions neuves et aux nouveaux décors peints évoqués durant la visite, souvent directement avec les artistes présents sur le chantier, pour les personnalités éminentes. Ainsi, le 26 août 1840, lors de la $201^{\mathrm{e}}$ visite, le Roi s'entretient "longuement dans la salle Constantine" avec le peintre Horace Vernet, avant de demander personnellement à Nepveu d'établir un devis de dorure ${ }^{13}$. Au-delà du simple compte rendu factuel, l'architecte estime les besoins financiers, aile par aile, pièce par pièce, qu'il déduit de chaque ordre émis par Louis-Philippe ; il y mêle nombre d'analyses personnelles sur la nature même des travaux à conduire et mentionne les visiteurs présents aux côtés du Roi, en général l'architecte et conseiller Pierre-Léonard Fontaine, le directeur général des musées royaux, Auguste de Forbin, puis Alphonse de Cailleux, directeur général des Beaux-Arts, proches du Roi, l'intendant général Montalivet...

En adressant son rapport à son supérieur hiérarchique, Nepveu traduit, au plan concret, les désirs royaux, décrit la méthode d'exécution, identifie les contraintes du site, choisit les corps de métiers à mobiliser, recommande des choix de matériaux et de techniques et livre les coûts estimatifs afférents à chaque poste. Parallèlement, il sollicite les entreprises pour établir un attachement, détaillant par le menu la réalisation matérielle, le temps d'intervention des journaliers, au moindre clou. Le fonds de l'agence d'architecture, au château de Versailles, conserve ainsi la plupart des mémoires d'entrepreneurs de 1832 à $1847^{14}$. La synthèse proposée par Nepveu, par ces rapports, a d'abord pour fonction de cerner les dépenses, pour solliciter les demandes de crédits au Trésor de la Couronne, un des services de la Liste civile. Dans le $92^{\mathrm{e}}$ rapport du 23 janvier 1837, il rappelle à Godard-Dubuc les « ordres directs du Roi " de rétablir un vestibule de l'escalier des Ambassadeurs, en lui demandant de débloquer les crédits nécessaires. À la lumière des rapports apparaît la complémentarité des deux hommes : l'un fait avancer le chantier, parfois au détriment de ses propres choix, pour se rapprocher de la volonté du souverain, quand l'autre veille à la dépense.

Sur la base des données de Nepveu, le directeur des Dépenses des bâtiments fait valider ou amender les sommes requises. Lors des visites des 9-30 novembre, Nepveu est ramené par le souverain à un projet relatif aux toitures du Grand Trianon. Dans une lettre complémentaire aux rapports de visite, l'architecte demande, le 18 novembre 1837, la réhabilitation de la couverture et le changement des plombs dans les combles du palais de Trianon; en réponse, le 27 novembre suivant, Godard-Dubuc précise à Nepveu qu'il a reçu du comte de Montalivet "l'approbation de la dépense des travaux qui comportai[en]t le rétablissement de la toiture (...) du Grand Trianon ${ }^{15}$. Godard-Dubuc reporte au crayon, en marge

13. Arch. nat., O/4/2858, 203 e rapport du 27 août 1840.

14. Arch. château Versailles, boîtes 1831/1 à 1847-22. Les 247 cartons, classés chronologiquement de 1830 à 1849 , contiennent correspondance, pièces de suivi comptable et budgétaire et mémoires des fournisseurs et entrepreneurs.

15. Arch. château Versailles, boîtes 1831-1 à 1837-1, nº 261. 
des rapports concernés, la somme réellement allouée. L'attention de Nepveu est soutenue : il défend assidûment son budget d'exécution.

Portées en marge du texte, les corrections chiffrées - plus de 200 annotations au crayon au total - sont nombreuses dans l'exemplaire des Archives nationales, beaucoup plus rares dans celui de Versailles. Lorsque Nepveu détaille une réalisation, ces chiffres précisent la somme attribuée et l'exercice budgétaire d'imputation. Le $\sigma^{\mathrm{e}}$ rapport du 18 janvier 1834, point $\mathrm{n}^{\mathrm{o}} 2$, évoque " l'établissement de la grande salle du rez-de-chaussée ", pour laquelle Godard-Dubuc attribue un "fonds de 60000 francs (1834)» en marge du texte. Dans le même rapport, une démolition imprévue oblige à recourir à un fond de réserve pour "l'évacuation des matériaux ". Le directeur attribue à Nepveu la somme de 20000 francs et déduit, en bon gestionnaire, le produit de la vente des matériaux.

Dans l'exemplaire des Archives nationales, des commentaires de Godard-Dubuc sont portés, au crayon, en marge du texte de Nepveu. Or, quelques-unes de ces annotations ultérieures se trouvent intégrées dans le corps même du texte des rapports de l'exemplaire de Versailles. Ainsi, lors de la $20^{\mathrm{e}}$ visite (page 118), le terme "royale », ajouté au-dessus de la ligne de texte de l'exemplaire des Archives nationales, a été incorporé au texte du rapport de la $20^{\mathrm{e}}$ visite dans l'exemplaire de Versailles. Ce pourrait fournir un indice définitif de l'ordre d'établissement de ces

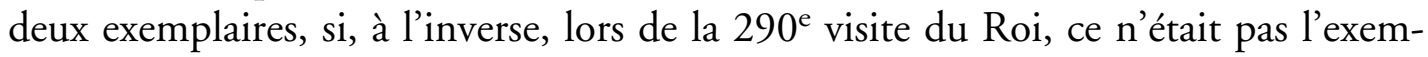
plaire de Versailles qui se trouvait amendé en marge (mentions : "au Roi», "la salle ", "même au plus heureux") et si ces corrections n'avaient pas été recopiées dans le texte de la version des Archives nationales, tout comme, d'ailleurs, dans l'exemplaire de Chantilly. Mais il est vrai que le cas reste tout à fait isolé. De façon générale, l'exemplaire de Chantilly est celui qui porte le moins d'annotations marginales de ce type (quatorze notes de marge en tout, et presque uniquement chiffrées) et même la correction "Louis XVI " en "Louis XIV ", que l'on note au bas de la première page de la $3^{\mathrm{e}}$ visite $\mathrm{du}$ Roi, est tout à fait accessoire ; sans doute l'architecte a-t-il relevé une coquille ponctuelle.

À l'analyse comparative de ces corrections, on pourrait donc conclure sommairement par le fait que les rapports de l'exemplaire des Archives nationales, signés de Nepveu et reçus par la Liste civile, sont les lettres originales, que différents intervenants (Godard-Dubuc et les collaborateurs des services comptables de l'Intendance) viennent annoter a posteriori, tandis que les rapports conservés par l'agence (Versailles) et par l'architecte à titre personnel (Chantilly) seraient établis sur la base des minutes préparatoires, mises au net ultérieurement en intégrant les annotations ajoutées par l'Administration, afin de disposer, pour mémoire, de la version la plus complète, la plus à jour et la plus conforme possible. Il est plus difficile d'affirmer laquelle est ensuite, d'un point de vue chronologique, entre Versailles et Chantilly, la première des deux mises au net : peut-être celle de l'agence précèdet-elle celle de l'architecte, ce qui expliquerait que ce dernier exemplaire (Chantilly) soit le moins chargé en annotations marginales.

Ces deux copies de travail reflètent, en tout état de cause, un travail minutieux et maintenu sur le temps long de collation des informations à l'original annoté. 
L'histoire et le goût de la collection sont à l'origine de canaux de transmission différents, entre sphère privée et sphère publique, jusqu'aux lieux de conservation actuels, tandis que la reliure de chacune des trois séries a figé en un état abusivement clos trois entités archivistiques. Mais elles ne tirent, en réalité, leur existence que d'un travail originel de rapprochement des textes.

\section{Intérêt des plans et croquis associés aux rapports de visites}

Sans évoquer les croquis à la plume portés en marge, les 47 documents figurés hors texte des quatre volumes des Archives nationales, reliés au milieu ou à la suite des rapports, sont, à l'exception des derniers feuillets du quatrième volume, en relation directe avec la teneur des rapports. Ces documents figurés se multiplient à partir du troisième volume, celui des années 1841-1843, quand Frédéric Nepveu semble précisément de plus en plus désireux de valoriser ses réalisations de maître d'œuvre: dans son rapport du 14 février 1842, l'architecte demande au Roi de l'autoriser à consacrer une salle à l'exposition de quarante dessins de sa création (ill. 1).

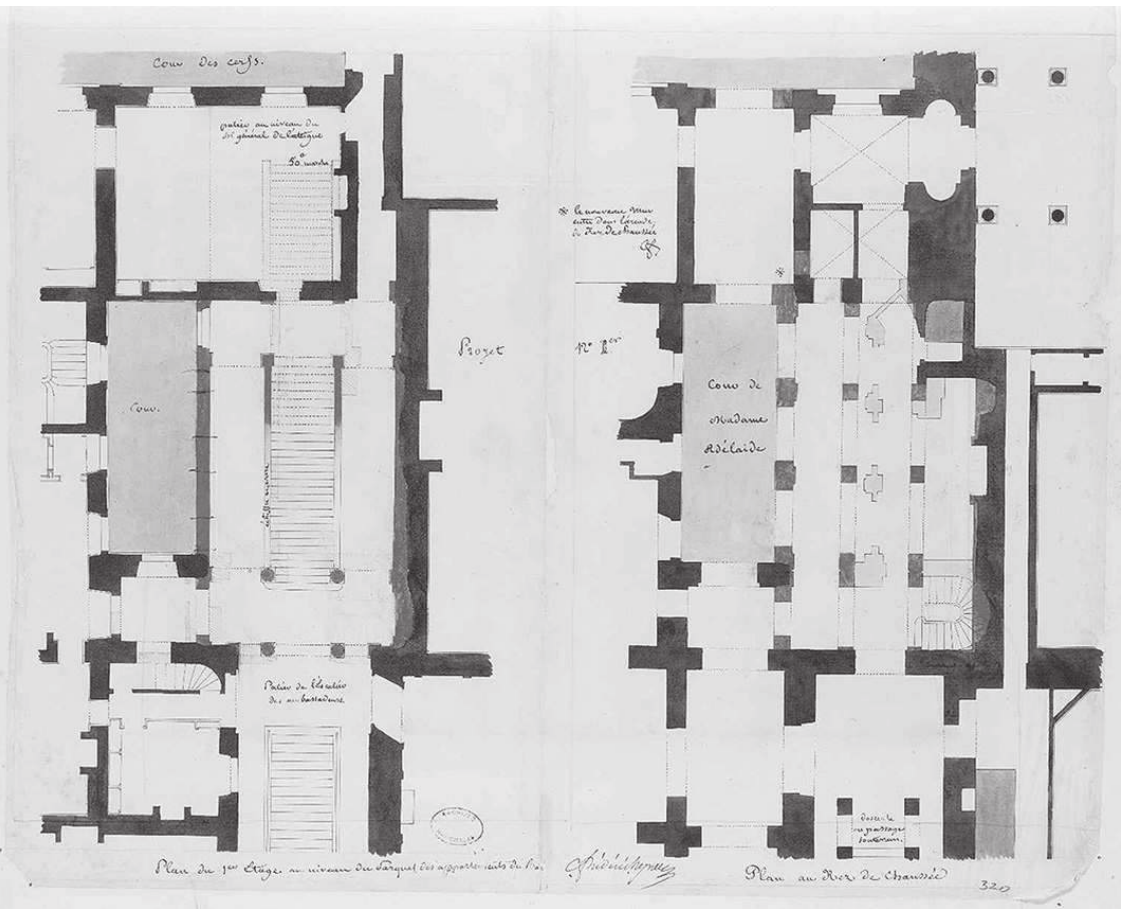

Ill. 1 : Projet de desserte de l'attique des grands appartements du Roi depuis le palier supérieur de l'escalier des Ambassadeurs, signé par Frédéric Nepveu, encre et lavis en couleur sur papier transparent, joint au rapport de la $299^{\mathrm{e}}$ visite du Roi du 16 novembre 1843. Arch. nat., O/4/2859. () Arch. nat.

Réalisés sur papier transparent (calque ou papier huilé), sauf exception, ces documents figurés sont constitués de plans, coupes et élévations, avec ou sans retombes, de croquis et de rares esquisses de projets de piédestaux et d'éléments sculptés. L'exemplaire de Chantilly propose 43 documents figurés, dessinés ou lavés, de la main de l'architecte, souvent en marge du texte (escalier de la chapelle, 
salle des Maréchaux, pavillon du Roi, grandes cuisines, plans de l'opéra, escalier des Généraux, salle des Académiciens).

Phase importante dans la formalisation des projets, l'architecte Nepveu dessine, avec son équipe, différents niveaux de plans, pour son propre compte, ou pour servir sur le chantier aux différents entrepreneurs retenus. Après l'inauguration de 1837, et l'édition par Charles Gavard d'un ouvrage de synthèse illustré de gravures, Nepveu propose à l'occasion, en annexe de tel ou tel rapport, le plan général gravé du château, tiré de cet ouvrage, pour localiser, à l'encre rouge, les zones des travaux en projet, de façon très synthétique, comme pour faciliter la compréhension des multiples lecteurs de ses rapports et donner à saisir le programme d'ensemble.

Les croquis en marge des rapports correspondent plutôt à des relevés d'intention: Nepveu jette l'idée rapidement à l'encre noire et rouge pour clarifier son propos. Dans le rapport du 13 juillet 1841, un schéma signé "Cf " (initiales de Charles Frédéric [Nepveu]) donne à voir, en quelques traits noirs seulement, le nom et les élévations des combles du château. Le coût estimé et la désignation des murs à déplacer sont indiqués à l'encre rouge dans les pièces dessinées. Il s'agit de faire comprendre que Louis-Philippe souhaite faire réaliser un "passage à plein pied à travers les combles au moyen d'une tour ronde, à l'angle nord-est de la salle du Sacre ", au croisement de l'attique de la salle de 1792, de la vieille aile et des appartements de la Reine ${ }^{16}$.

Les plans des salles du musée à créer sont quasiment les mêmes, pour le contenu, entre l'exemplaire de Chantilly et celui des Archives nationales, mais les documents figurés insérés dans l'exemplaire personnel de Nepveu (Chantilly) sont minutieusement travaillés, alors que les plans adressés à l'administration centrale (Archives nationales) comprennent plus d'éléments techniques, coûts par poste et notes (noms des entreprises écrits à la main dans les salles). Ainsi, les plans de l'opéra construit sous Louis XV, de grande qualité dans l'exemplaire de Chantilly, sont enrichis, pour Godard Dubuc, de la main de Nepveu, des quelques décors neufs souhaités (dorure, peinture) (ill. 2).

De l'ébauche, plus ou moins soignée, Nepveu aboutit le plus souvent à un plan descriptif détaillé, à l'encre noire et lavis polychrome, et de plus grand format. Pour un même lieu, la documentation multiple (plans, coupes, élévations) permet de lire les transformations engagées : dans le plan certifié du 5 avril 1844, sans doute destiné aussi à l'entreprise versaillaise de charpente de Jean-Michel Touchard, Nepveu isole au lavis rose l'épure des voussures du grand salon d'Hercule (ill. 3) marquant les élévations à modifier et légendant avec précision les différentes zones.

Certains de ces plans sur papier transparent, sans avoir la qualité d'exécution des plans lavés sur papier conservés dans le fonds d'agence, peuvent, telle cette coupe de la jonction de l'aile du Midi et du pavillon d'Orléans (ill. 4), revendiquer une vraie efficacité esthétique, quand l'enjeu de la précision amène toutefois l'architecte à de copieuses annotations explicatives à l'encre rouge.

16. Arch. nat., O/4/2858, dessin à l'appui du $221^{\mathrm{e}}$ rapport du 13 juillet 1841 . 


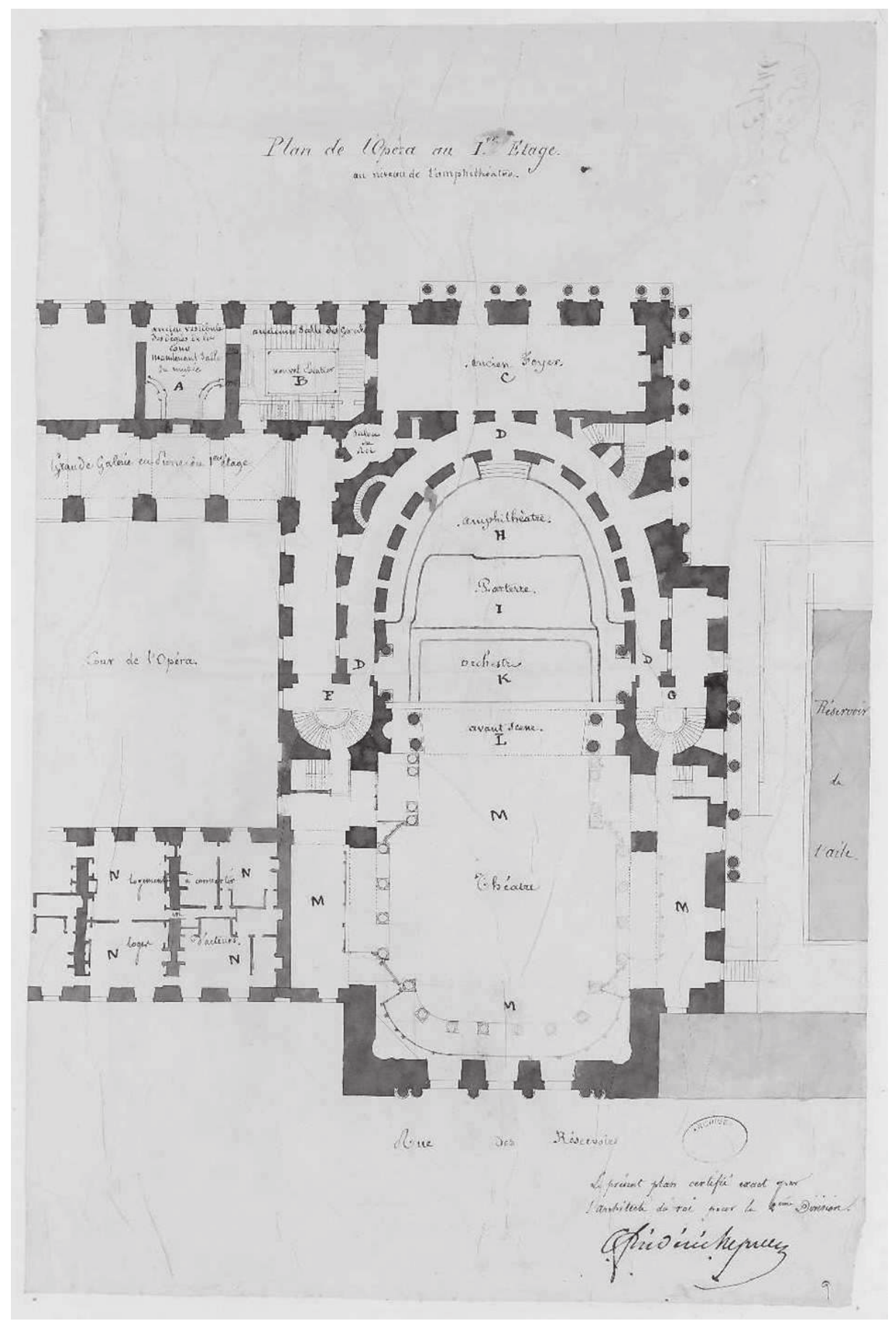

Ill. 2: "Plan de l'Opéra au $1^{\text {er }}$ étage/au niveau de l'amphithéâtre ", certifié et signé par Frédéric Nepveu, architecte du Roi pour la $2^{\mathrm{e}}$ division, encre brune et lavis sur papier transparent, $39 \times 25 \mathrm{~cm}$, joint au rapport sur la $89^{\mathrm{e}}$ visite du Roi du 4 février 1837. Arch. nat., O/4/2858. (C) Arch. nat.

Dans leur ensemble, les 47 documents figurés de l'exemplaire des Archives nationales sont signés "Frédéric Nepveu », mais sont plus vraisemblablement l'œuvre des dessinateurs de son agence, dont nous savons qu'elle put être composée de plus de 160 membres. Validant le travail de son équipe par son certificat et sa signature, il écrit parfois directement sur la feuille, au milieu d'une salle, les réalisations 


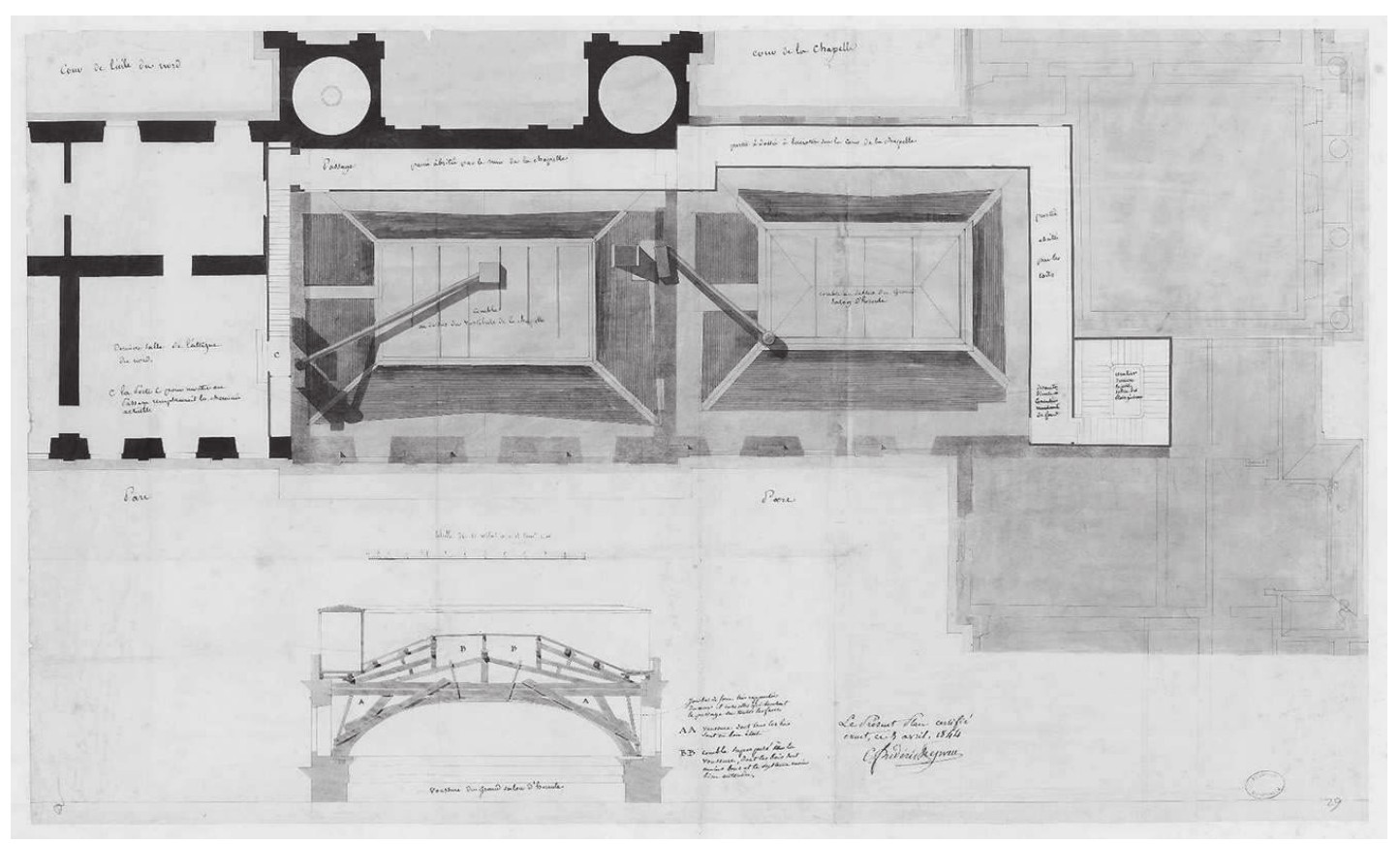

Ill. 3 : Plan des toitures des combles du vestibule de la chapelle et du grand salon d'Hercule et coupe de la voussure du grand salon d'Hercule, certifié et signé par Frédéric Nepveu, 3 avril 1844, encre noire et rouge et lavis en couleur sur papier transparent, $44 \times 66 \mathrm{~cm}$, à l'appui du $310^{\mathrm{e}}$ rapport du 2 avril 1844. Arch. nat., O/4/2860. (C) Arch. nat.

demandées aux entrepreneurs : il lui arrive ainsi de comparer le prix de travaux de dorure et d'en fixer le prix définitif, en inscrivant clairement sa décision sur le plan concerné (ill. 5).

Parmi ces ajouts de la main de Nepveu figurent aussi des mentions fonctionnelles, tel que le déplacement d'un calorifere préalable à la modification d'un espace : la décision technique est matérialisée d'une croix sur le plan adressé à Godard-Dubuc (ill. 6).

L'attention aux détails a peu de limites : ici, il décrit le décor à exécuter à la peinture à l'huile, pour l'encadrement des motifs de bas-reliefs en stuc d'un escalier, livrant précisément le dessin des trophées à reproduire; là, il demande à rehausser tel socle de statue (ill. 7).

En ses rapports, Nepveu écrit fréquemment qu'il ne décide pas seul. En proposant un projet figuré, il attend aussi la validation d'un parti pris (implantation de murs de cloisons, thème ornemental...) et abandonne peut-être une part du principe de la décision à $\mathrm{O}$. Godard-Dubuc, sinon au Roi même (ill. 8).

Ces 47 documents figurés hors texte, majoritairement sur papier transparent, associés aux rapports de l'exemplaire de l'Intendance de la Liste civile, n'existent pas par ailleurs aux Archives nationales sous d'autres formes (originaux sur support papier ou copies sur calque ou papier huilé), ni en sous-série $\mathrm{O} / 4$, ni en sous-série F/21 (Beaux-Arts), ni en série N (Cartes, plans et dessins d'architecture). Dans le versement de la direction de l'Architecture (série VA) sont conservés en effet douze plans signés de Frédéric Nepveu pour des travaux à l'aile du Midi du château de 


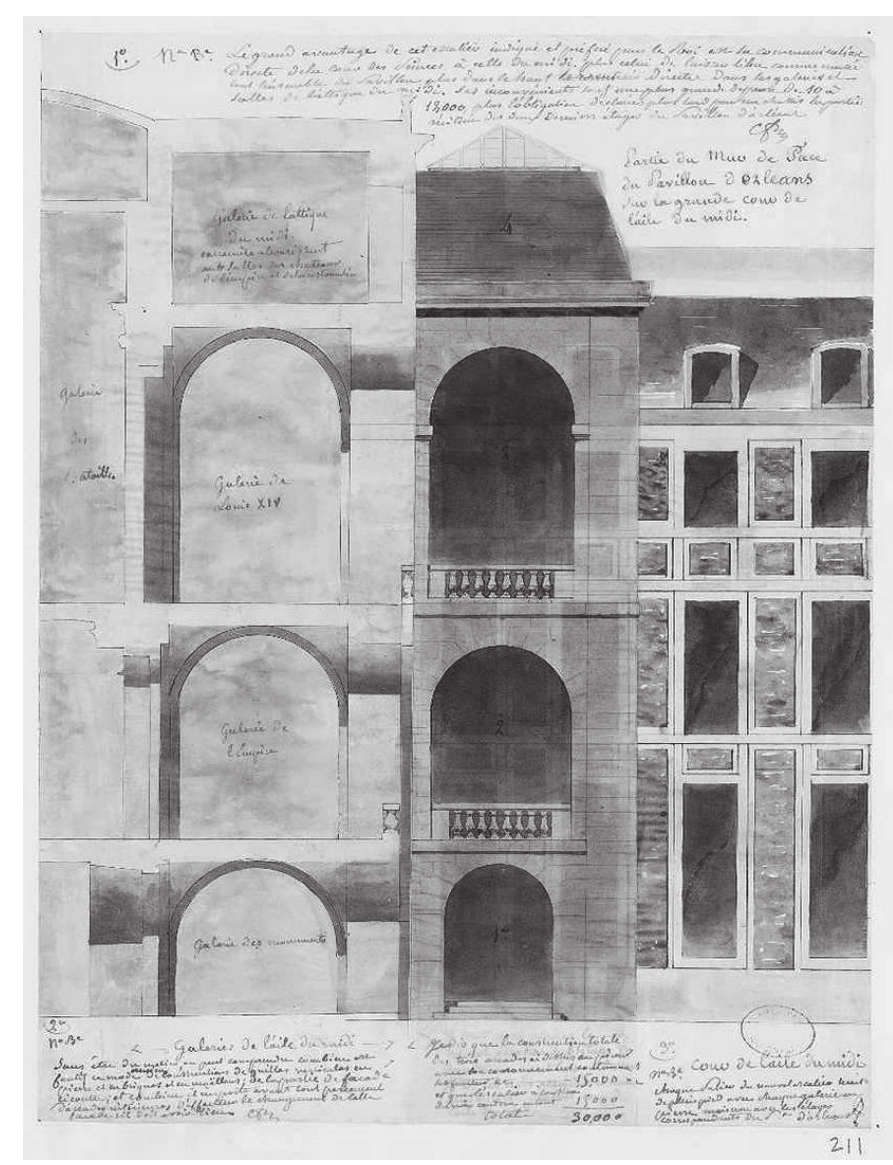

Ill. 4 : Élévation du pavillon d'Orléans sur la cour de l'aile du Midi et coupe des galeries de l'aile du Midi, signé par Frédéric Nepveu, encre brune et rouge et lavis en couleur sur papier transparent, 29,5 × $23 \mathrm{~cm}$, à l'appui du rapport de la $361^{\mathrm{e}}$ visite du 11 avril 1846. Arch. nat., O/4/2860. () Arch. nat.

Versailles ${ }^{17}$, datés des années 1833-1834, dont dix sont assurément liés à l'élaboration de rapports au directeur des Bâtiments de la Couronne des 19 août et 25 septembre 1833. Sur papier transparent également, ils ne sont pas redondants avec ceux des volumes reliés et constituent sans doute, eux aussi, des copies (un seul porte un numéro d'enregistrement) d'originaux sur papier conservés dans le fonds de l'agence d'architecture de Versailles.

En effet, c'est bien dans ce fonds versaillais, riche de 1850 plans et croquis de tous formats (coupes, élévations, plans, dessins de décors monumentaux, croquis d'éléments sculptés et de pièces techniques (caloriferes), esquisses de moulures, tracés de conduites d'eau...), qu'est regroupée la documentation graphique la plus complète sur le chantier du musée de Versailles, à la disposition du maître d'œuvre comme de tous les corps de métier, pour définir le temps de travail, acquérir les matériaux en quantité suffisante, préciser toutes les mesures et dimensions, jusqu'au moindre miroir du versaillais Fisanne, - à la mesure donc d'un des plus grands chantiers du règne de Louis-Philippe.

17. Arch. nat., VA 29, plans 31 à 42. Le détail en est donné dans les sources complémentaires de l'inventaire en ligne, déjà cité, des registres Montalivet. 


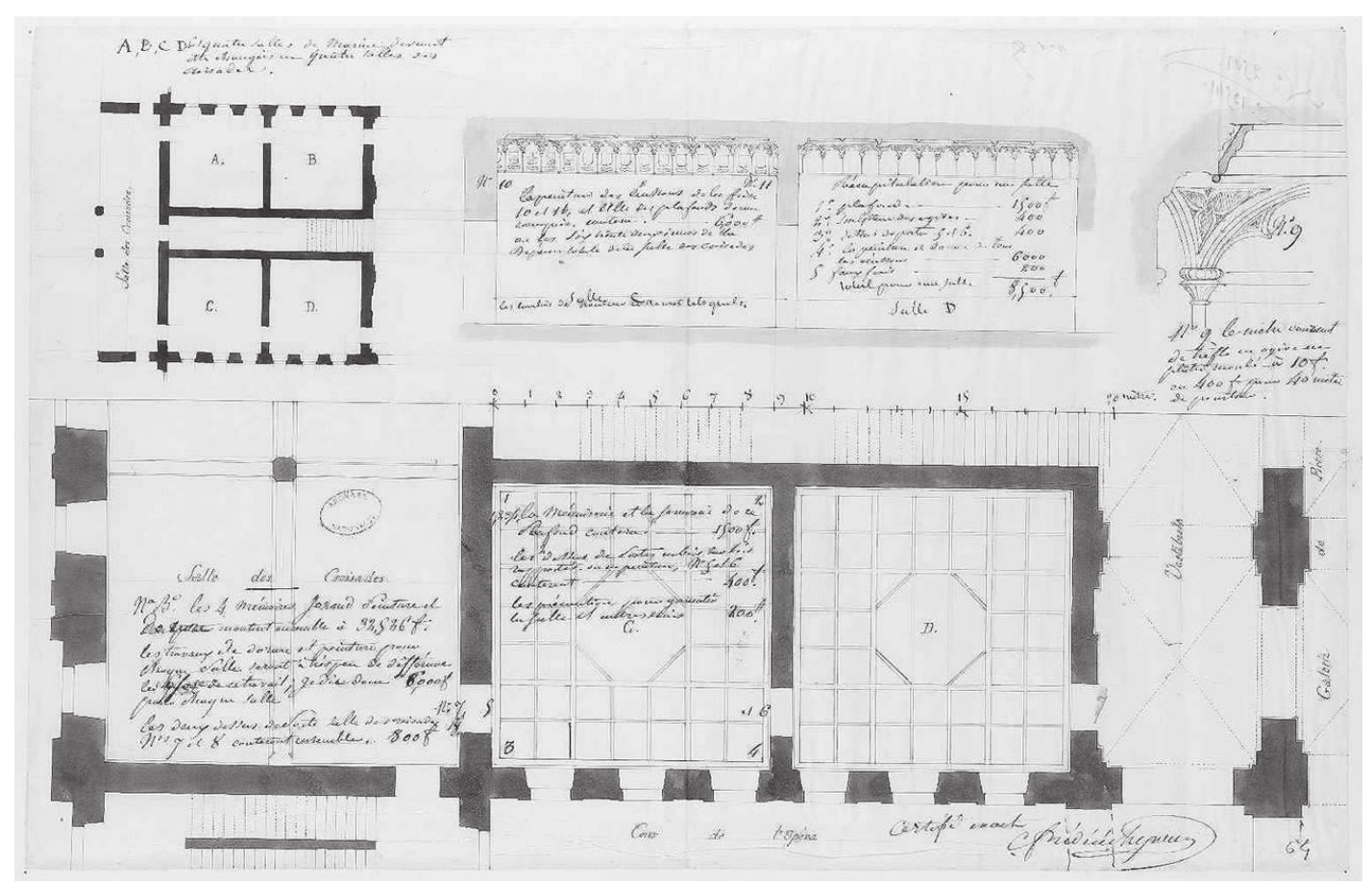

Ill. 5 : Plans, coupe et détail des nouvelles salles des Croisades, certifié et signé par Frédéric Nepveu, encre brune, encre rouge et lavis rose sur papier transparent, $27 \times 42 \mathrm{~cm}$, joint au rapport de la $239^{\mathrm{e}}$ visite du Roi du 30 mars 1842. Arch. nat., O/4/2859. (C) Arch. nat.

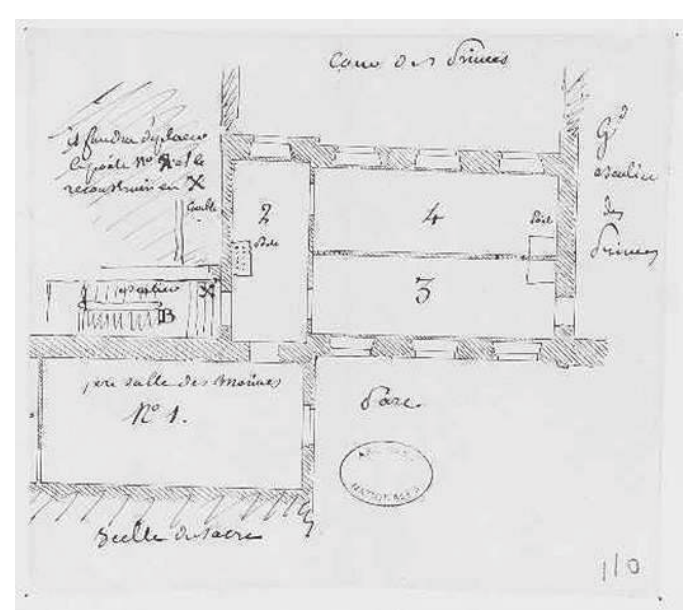

Ill. 6: Croquis des salles projetées entre la salle du Sacre et le grand escalier des Princes, [Frédéric Nepveu], encre brune sur papier transparent, $12 \times 13,5 \mathrm{~cm}$, joint au rapport de la $252^{\mathrm{e}}$ visite du Roi du 21 octobre 1842. Arch. nat., O/4/2859. () Arch. nat.

\section{Le reflet d'une chaîne administrative complexe}

\section{La réception des rapports de Nepveu à l'Intendance générale}

Le rapport de visite signé par Frédéric Nepveu est le plus souvent rédigé le jour même du passage du Roi, parfois le lendemain. Un délai de deux à quinze jours est à noter parfois, en période hivernale ou lors des rares absences de l'architecte. 


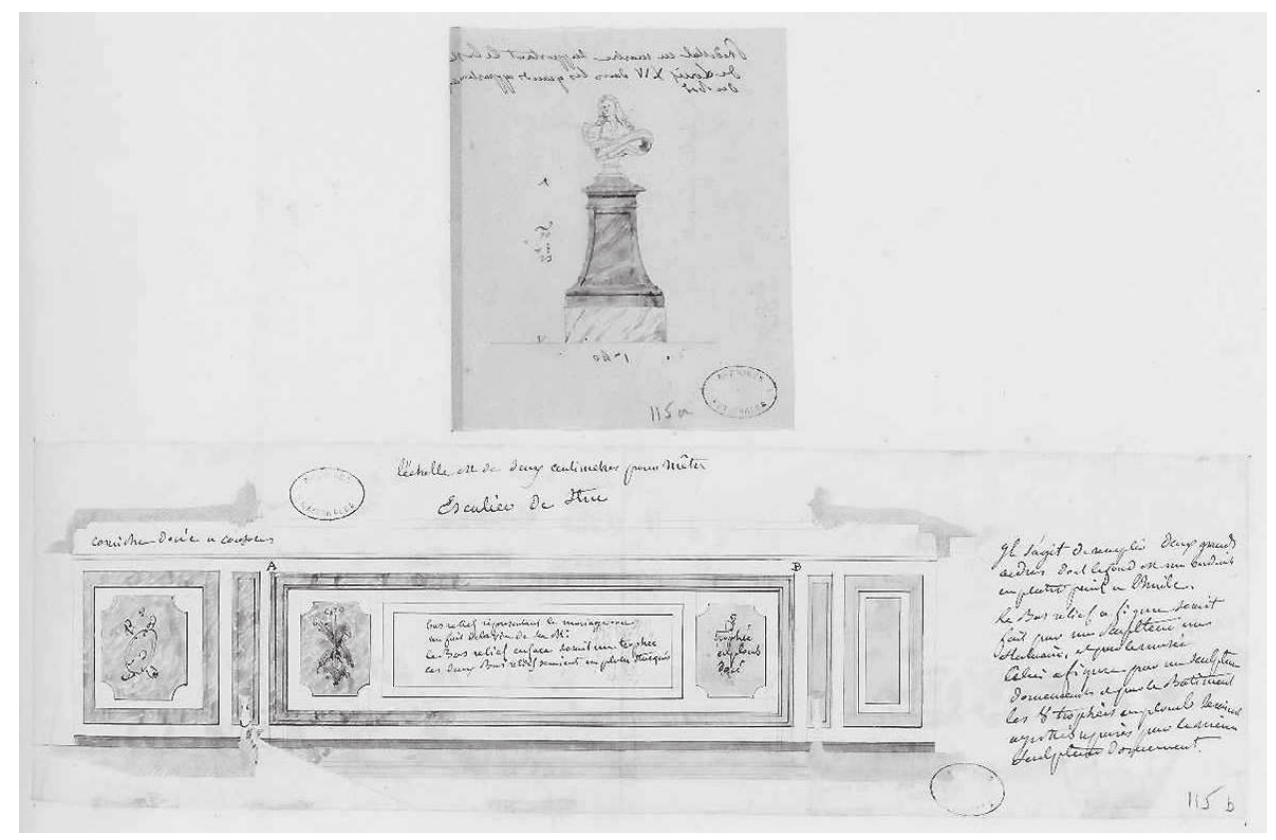

Ill. 7 : Projet de piédestal pour le buste de Louis XIV des grands appartements du Roi, encre brune et lavis de couleur sur papier transparent, haut. $11 \times \operatorname{larg} .9 \mathrm{~cm}$, et Projet de bas-relief pour l'escalier de stuc, encre brune et lavis de couleur sur papier, $9,5 \times 33,5 \mathrm{~cm}$, dessins par [Frédéric Nepveu] joints au rapport de la $253^{\mathrm{e}}$ visite du Roi du 28 octobre 1842. Arch. nat., O/4/2859. (C) Arch. nat.

Le destinataire du rapport, le directeur des Dépenses des bâtiments de la Couronne, Godard-Dubuc, dépendait hiérarchiquement de l'intendant général de la Liste civile. En cas d'absence prolongée de Nepveu, l'intérim de son poste, notamment pour la rédaction de ces rapports de visites, est assuré par un inspecteur du service des Bâtiments, Mondel, comme ce fut le cas de mai à septembre 1839. Lorsque Godard-Dubuc s'absente, les rapports sont adressés directement à l'intendant général de la Liste civile : le comte de Bondy, pour l'intérim de mars 1838mars 1839, ou le comte de Montalivet, pour celui d'avril 1839.

Les rapports de Nepveu, ainsi que, parfois, les plans associés, sont pourvus d'un numéro d'expédition, variable; d'un numéro unique («2501»), porté sur l'ensemble des lettres; d'un numéro d'enregistrement à l'arrivée; d'apostilles marginales, au crayon ou à l'encre, essentiellement relatives aux conséquences financières et comptables des ordres de travaux donnés par le Roi (" Un crédit de 190000 francs est affecté aux travaux dont il est question ", dans le rapport du 41833 ; "Fonds de 60000 francs ", dans le rapport du 18 janvier $1834 \ldots$..) ; enfin, de renvois aux différents bureaux de l'Intendance générale.

Le numéro d'expédition de l'agence de Nepveu, à l'encre noire, est rédigé de la même main que le texte ; il n'est pas apposé systématiquement et ne suit pas un ordre précis, pour une raison encore indéterminée. Les deux exemplaires du château de Chantilly (à l'usage de Nepveu) et des Archives nationales (exemplaire de Montalivet) présentent les mêmes numéros d'expédition. Le numéro " 2501 ", commun à tous les rapports jusqu'en 1847, identifie précisément la correspondance du chantier du château de Versailles, sous l'appellation "Restauration du Palais ». 


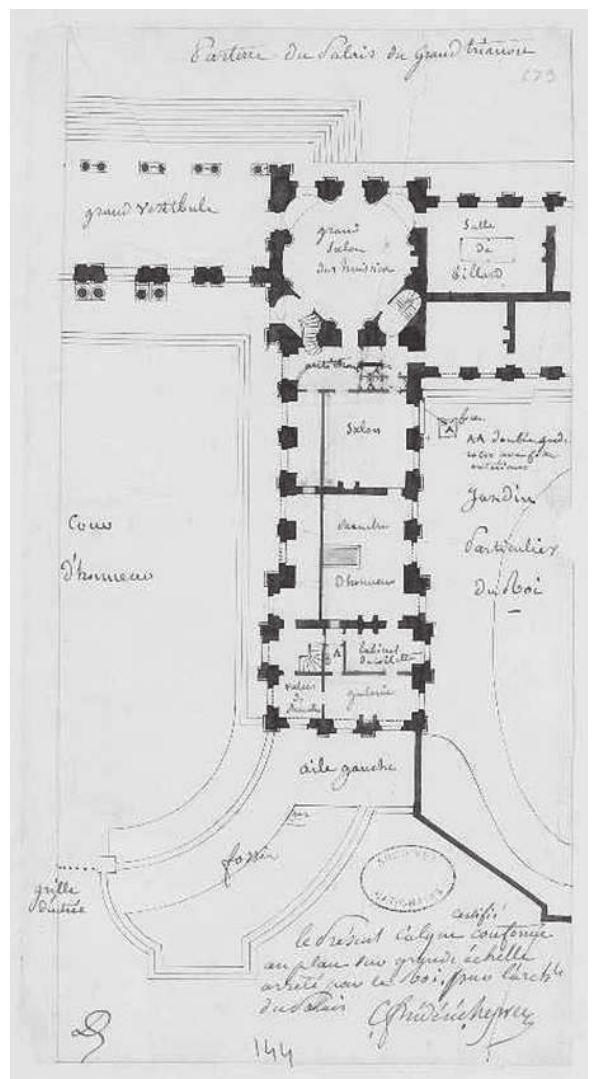

Ill. 8 : Plan d'aménagement d'appartements dans une aile du palais du Grand Trianon, certifié et signé par Frédéric Nepveu, architecte du Palais, encre noire et rouge et lavis en couleur sur papier transparent, $21,5 \times 11,5 \mathrm{~cm}$, joint au rapport de la $340^{\mathrm{e}}$ visite du Roi du 7 juillet 1845 . Arch. nat., O/4/2860. (C) Arch. nat.

Ce numéro rouge est suivi d'une numérotation en noir, précédée d'un « $\mathrm{E}$ », qui signifie l'enregistrement des rapports à leur arrivée au secrétariat de la Liste civile. Les quatre registres présentent une numérotation d'enregistrement croissante, mais non consécutive, laissant à penser que ces rapports de Nepveu sont intégrés à l'enregistrement global des lettres reçues par la direction des Dépenses des bâtiments durant le règne.

Les mentions marginales peuvent signaler les suites données aux rapports de Nepveu ("Le 29 août 1833, écrit à M. Nepveu ", ou "Répondu le 15 février $1834 »)$. Durant l'intérim du comte de Bondy, les rapports portent en outre le large cachet rouge de son cabinet particulier. Les mentions occasionnelles (" rapport au Roi du...»), portées en marge, ou encore l'ordre ajouté au feuillet $\mathrm{du}$ 12 février 1840 ( $M$. Dubuc. Faire un Rapport au roi sans conclusion, en lui demandant ses ordres ») indiquent que le Louis-Philippe ne lisait pas systématiquement les rapports de l'architecte dans leur version extensive, mais qu'une synthèse pouvait, si nécessaire, lui en être présentée. Lors d'une visite ultérieure, il arrive à Louis-Philippe de constater expressément la mise en œuvre des transformations commandées, demandant parfois des modifications supplémentaires. 


\section{Un témoignage de premier ordre sur le processus administratif et comptable} des services de la Liste civile

L'administration de la Liste civile, créée par la loi du 2 mars 1832, après un long débat parlementaire, reçut la plupart des multiples attributions de l'ancienne Maison du Roi de Louis XVIII et Charles X, dont la gestion des bâtiments de la Couronne. Ses services disposaient des crédits relevant du budget voté par la Nation, fonds mis à disposition du souverain et de l'Intendance générale qui les gère, afin de permettre au souverain de tenir son rang en entretenant ses résidences et en faisant fonctionner la Cour. Ce principe prit fin par décret du 26 février 1848, lorsque le Gouvernement provisoire rendit à la gestion ordinaire des biens de l'État l'ensemble des services dépendant de la Liste civile, décret confirmé par un décret de l'Assemblée nationale, le 25 octobre suivant.

Dans ce cadre législatif, le Roi apporte aux Domaines de la Couronne les changements utiles à leur conservation et embellissement (art. 14), tandis que l'entretien et les réparations de ces domaines, pour lesquels le Roi reçoit chaque année du Trésor public une somme de 12 millions (art. 17), étaient expressément à la charge de la Liste civile (art. 15). S'y ajoutait le million attribué à son aîné, le princehéritier Ferdinand. Le domaine de Versailles fait partie de la dotation immobilière dont peut jouir le Roi (art. 2), comprenant en tout onze palais, trois manufactures et trois forêts, ainsi que l'apanage direct des Orléans. La dotation mobilière englobe les pierreries, statues, tableaux et meubles des palais royaux et du Garde-Meuble de la Couronne, à faire restaurer par des doreurs, sculpteurs ou spécialistes employés aux frais de la Liste civile.

L'organigramme se fixe à partir de la seconde moitié de 1832 : dirigée par un Intendant général, la Liste civile est organisée en trois pôles, déclinés en dix-sept directions. Le pôle Commandement (1) s'articule autour de l'Intendance générale, du cabinet de l'Intendant général, de la division Centrale, du Conseil et de l'intendant général honoraire. Le pôle Comptable (2) englobe le Trésor de la Couronne, la direction de la Comptabilité générale, la direction des Dépenses des bâtiments, la conservation du Mobilier de la Couronne, la conservation des Forêts de la Couronne, le conseil de la Liste civile et la direction des Domaines et du contentieux. Le pôle Arts, lettres et manufactures (3) embrasse la direction des Musées royaux, la direction des Manufactures royales (Sèvres, Gobelins, Beauvais) et la direction des Bibliothèques de la Couronne. À sa tête durant douze des dix-sept années du règne orléaniste, Montalivet, polytechnicien et ingénieur, était un libéral convaincu, bouc émissaire des caricaturistes et opposants qui ne cessèrent de vilipender cette administration dépensière, incontrôlable et inutile.

Installée à l'hôtel du Grand-Veneur, 9 place Vendôme, l'Intendance générale de la Liste civile, étudiée par David Frapet précisément pour son rôle dans la commande monumentale ${ }^{18}$, intervient dans la gestion comptable, le contrôle des

18. David Frapet, "Les politiques publiques conduites en faveur des monuments français sous la monarchie de Juillet par le Parlement et la Liste civile ", thèse d'histoire sous la direction de David Deroussin, soutenue à l'université Jean-Moulin de Lyon le 17 décembre 2012 [en ligne: http:// 
dépenses, le déblocage des fonds et la validation des mémoires tout à la fois, hors de tout contrôle externe de la Cour des comptes, du Parlement et de l'électeur. Le Roi y emploie toutefois des juristes et des contrôleurs de la dépense, qui la rationalisent et en garantissent les missions principales. Dans les archives de l'agence d'architecture de Versailles, de multiples corrections, sous la forme de rayures en rouge, sont du reste directement apposées par des contrôleurs sur les chiffres des attachements et mémoires des entreprises et fournisseurs employés sur le chantier, témoignant de la mise en œuvre de ce contrôle interne. Si l'Intendance générale était une entreprise privée, le Roi serait le "président" de la Liste civile, décidant jusqu'aux moindres détails du chantier, selon l'image donnée par les rapports de Nepveu de 1833 à 1847.

Les travaux aux bâtiments royaux, théoriquement soumis à l'approbation du souverain, devaient, en pratique, être approuvés par l'Intendance générale, qui le représente et qui les finance. Godard-Dubuc recevait ainsi les lettres-rapports de Nepveu sur les visites et décisions de Louis-Philippe à Versailles, pour les transmettre à l'Intendant général, afin que celui-ci donne son avis et son aval administratif au lancement et au financement des travaux. La direction des Dépenses des bâtiments de la Couronne constitue une pièce maittresse dans la mise en œuvre des politiques artistique et architecturale. Malgré la perte quasi intégrale des archives de cette direction technique, les mandats de paiement conservés via la série budgétaire de l'Intendance générale ${ }^{19}$ et l'Almanach royal et national de 1833-1847 permettent d'en étudier l'organisation et le champ d'action. En 1832, quand Nepveu prend la charge du château de Versailles et que Godard-Dubuc entre en fonction aux Bâtiments de la Couronne, les deux protagonistes se connaissent déjà : Nepveu appartint au service des Palais, maisons et résidences royales à compter de 1824 ; Godard-Dubuc fut intendant militaire de la Liste civile sous le règne de Charles X. Au sein du pôle Comptable de l'Intendance générale, le directeur des Dépenses des Bâtiments était secondé par un comité consultatif de quatre architectes (Dubois, Huvé, Dufour et Gisors), afin d'estimer les devis présentés par les architectes en charge de chaque bâtiment, tandis que les mémoires étaient vérifiés par cinq employés du "bureau de vérification" de la direction.

La teneur des rapports de visite à Versailles laisse transparaître l'excellente connaissance, par Frédéric Nepveu, des ressources locales du secteur du bâtiment. De fait, c'est à lui que revient la question du recrutement et du choix des entrepreneurs et fournisseurs spécialisés. Si quelques-uns sont parisiens ou extérieurs ${ }^{20}$,

www.theses.fr/?q=Frapet+david]. Elle complète la thèse d'histoire du droit de Damien Salles, «La fortune du Prince au XIX ${ }^{e}$ siècle : étude sur la Liste civile en France ", sous la direction de JeanLouis Harouel, université Panthéon-Assas (Paris), 2008.

19. Arch. nat., O/4/1329-O/4/2375.

20. Ainsi Dubois, Jorand et Moensch (dorure) ; Feuchère et Fossey (bronzes) ; Plantar (sculpture) ; Abel de Pujol, Cicéri, Lefébure, Nourry, Saint-Denis et Vernet (peinture) ; Huber et Wallet (ornements) ; Alasia et Borrani ("poêlerie »); Crovatto (stucs); Bex, Grégory et Hersent (marbrerie) ; Gothreau, frotteur ; Mignon (serrurerie) ; Létang, fondeur ; Jacob Desmalter, Henry et Packham (menuiserie) ; Noguet (maçonnerie). 
le bassin d'emploi local est remarquablement valorisé : les marbriers Beaumont et Dunoyer, les peintres Cornué et Renaud, le vitrier Demonceaux-Montigny, le errassier-paveur Oblin, les maçons Fournier, Huret, Lefuel, Ouachée, Paris, Reculard et Vidal, le fondeur Amard, les serruriers Collard et Denis, les menuisiers Binart et Morel, les couvreurs Lambert Baudry et Fontaine (Charles), le plombier Fontaine (Constant), les carreleurs Pichot et Quéro, le doreur Lebeaux, le rampiste Duaille, le fumiste Bozzi, le marchand de bois Guilloteau-Vatel, le miroitier Fisanne, les marchands de papiers peints Pendret et Ralec, le quincailler Baujon, le charpentier Touchard, l'épicier Delaferrière, le cordier Lucas, le vitrier Baligand et le ferblantier Prémia ont tous leur entreprise localisée à Versailles ${ }^{21}$. Les entreprises signent des actes de soumission ${ }^{22}$ au règlement imposé, dont une clause fondamentale, accepter de subir la modification des sommes des attachements et mémoires édités et détaillés poste par poste. Le cheminement du paiement est très long, à l'examen des dates de chaque intermédiaire du contrôle comptable, signant les mémoires des entrepreneurs conservés dans le fonds d'agence à Versailles.

David Frapet a, de fait, identifié dans sa thèse huit niveaux d'intervention, dans le processus administratif, pour régler la dépense à l'artisan, à compter de la certification de l'inspection du travail fait, elle-même certifiée par l'architecte Nepveu, qui propose de régler la somme demandée. Le vérificateur-expert des bâtiments du Roi certifie à son tour, en révisant souvent l'ensemble des calculs. Le comité consultatif des Bâtiments examine et révise les chiffres consolidés des mémoires, le tout étant signé in fine par Godard-Dubuc à nouveau. Quant au paiement de l'artisan, il est rendu possible par un mandat de paiement émis par Godard-Dubuc, qui passe en signature dans les différents services comptables jusqu'à l'Intendant général même. Après plusieurs années parfois, l'entreprise reçoit un avis par courrier, bénéficiant d'un délai de cinq jours pour retirer la somme à la caisse du Trésor de la Couronne. Lenteurs et complexités sont sans doute un des arguments de la fidélité des entrepreneurs au chantier, dont rendent bien compte les rapports de Nepveu sur la longue durée, tout comme la documentation comptable conservée par ailleurs : s'assurer d'obtenir, par leur présence et leur visibilité, le paiement définitif de leurs travaux.

\section{Conclusion}

En l'absence de ministère dédié, c'est sur l'Intendance de la Liste civile, qui conservait évidemment les Beaux-Arts dans les prérogatives de la Couronne, que Louis-Philippe s'appuie pour mettre en place son projet de réhabilitation du château de Versailles, vide depuis la Révolution. Selon les rapports de Nepveu, notamment, ces travaux d'ampleur touchèrent le corps principal et les ailes du Nord et

21. Pour le détail des adresses respectives de ces entrepreneurs et fournisseurs locaux, relevées par David Frapet, voir l'inventaire en ligne, déjà cité, du fonds O/4/1329-O/4/2375 aux Archives nationales. 22. Arch. château Versailles, boîte « 1836 ", nº 23. 
du Midi, du sous-sol à la toiture, sans oublier les pavillons d'Orléans et le château du Grand Trianon. Si les transformations principales eurent lieu avant 1837, le Roi maintint ce lien privilégié jusqu'à la fin du règne, en supervisant de nouvelles restaurations et de nouveaux programmes décoratifs, à l'instar des des salles d'Afrique, inachevées. D'après Montalivet (1850), 33615095 francs de dépenses dites extraordinaires auront été investis par l'Intendance de la Liste civile dans les bâtiments de la Couronne, auxquels s'ajoutent 1560000 francs investis dans les parcs et jardins du domaine de la Couronne ${ }^{23}$. Quatre bâtiments (Versailles, Tuileries, Saint-Cloud et Fontainebleau) concentrent 74,4\% des dépenses relatives aux bâtiments de la Couronne de 1831 à février 1848, Versailles, le Grand et le Petit Trianon absorbant à eux seuls $36 \%$ du total des crédits. Encore peut-on penser cette estimation minimale: le député Vavin, liquidateur général de la Liste civile, avance une somme bien supérieure de 53029476 francs, y compris l'entretien ordinaire, dépensés dans les bâtiments de la Couronne par la Liste civile de Louis-Philippe ${ }^{24}$. Versailles est «improductif», put écrire l'avocat Philippe Dupin ${ }^{25}$, membre du Conseil de la Liste civile, réalité comptable au regard des recettes tirées des domaines et forêts de la Liste civile.

Mais quel pouvait être le prix d'un tel enjeu politique : réconcilier les partisans des différents régimes qui s'étaient succédé depuis 1789, asseoir la légitimité du roi de tous les Français, construire l'identité nationale, par la célébration des conquêtes militaires de l'Ancien Régime, de la Révolution française, de l'Empire et même de la Restauration? Quelle pouvait être la limite à l'intervention personnelle du souverain? Ces quatre "albums Montalivet " attestent à quel point le monarque en personne surveilla l'exécution des travaux et les programmes, discutant le plan de toutes les salles et les partis décoratifs. Les rapports de Nepveu, émanation des décisions du Roi, reçurent ainsi globalement un écho favorable 9 place Vendôme. Pourtant le riche volume illustré publié à l'occasion de l'inauguration de 1837, en présence des grands corps de l'État, de l'Institut et du monde des lettres et des arts, malgré de nombreuses gravures de la Galerie royale, fait bien peu de cas de la production figurée de l'architecte Nepveu ${ }^{26}$.

Attaché au symbole monumental du pouvoir monarchique, Louis-Philippe livra aux visiteurs et aux électeurs, plus qu'une simple restauration, une transformation d'envergure, faisant en sorte "que les monuments de toutes nos gloires nationales" fussent "environnés ainsi de la magnificence de Louis XIV » ${ }^{27}$. Par la voie la plus

23. Camille de Montalivet, "Le roi Louis-Philippe et sa Liste civile ", La Revue des Deux-Mondes, t. 8, 1850 ; rééd. Paris, Michel Lévy frères, 1851.

24. Alexis Vavin, Compte de la liquidation de la Liste civile et du domaine privé du roi Louis-Philippe, rendu le 30 décembre 1851, Paris, Charles Noblet, 1852.

25. Moniteur universel, 10 janvier 1832, p. 83.

26. Charles Gavard, Galeries historiques de Versailles, publiées par ordre du Roi et dédiées à S. M. la reine des Français, Paris, chez l'éditeur et au Palais de Versailles, 1837.

27. Cité par Thomas W. Gaehtgens, Versailles de la résidence au Musée historique. La galerie des Batailles dans le musée historique de Louis-Philippe, Anvers, Fonds Mercator, 1984 [rééd. Paris, Albin Michel, 1992], p. 64. 
matérielle et technique qui soit, les registres de Nepveu font entrer dans les coulisses d'un scénario dont nous connaissons le terme : à Versailles, en un peu plus d'une décennie, Louis-Philippe s'est affirmé comme l'un des souverains bâtisseurs les plus importants du XIX ${ }^{\mathrm{e}}$ siècle.

Isabelle CHAVE responsable du département de l'Exécutif et du Législatif aux Archives nationales Éric LANDGRAF doctorant de l'université de Paris-Saclay 